\title{
Discusión con Luis Villoro. ¿Es para los modernos la libertad el único contenido posible del bien común?
}

\author{
CARLOS PEREDA \\ Instituto de Investigaciones Filosoficas, UNAM
}

La vasta, siempre lúcida y no pocas veces penetradora obra de Luis Villoro es ejemplar por lo menos en dos sentidos de esta palabra. En primer lugar, es ejemplar en sí misma: una de las obras de pensamiento que las generaciones próximas de América Latina podrán interrogar buscando valores genuinamente filosóficos y no el mero documento histórico. Me interesa subrayar este punto: sin duda Villoro ha estado toda su vida preocupado por su entorno histórico-social; al respecto recuerdo dos libros suyos que se encuentran ya entre nuestros clásicos: El proceso ideológico de la revolución de la Independencia y $L o s$ grandes momentos del indigenismo en México, pero no sólo; en relación con esta honda preocupación por su circunstancia sería una torpeza pasar por alto sus agudísimos artículos de opinión, que han abordado desde temas como el aborto hasta discusiones sobre el EZLN y los problemas actuales del multiculturismo, sobre todo en relación con los pueblos indigenas. Sin embargo, no nos confundamos: la obra de Villoro, aunque generosamente comprometida, o más bien, precisamente por ello, nunca sucumbe al vicio del entusiasmo nacionalista, y sin duda rehuye por completo el color local; de ahí que en ningún momento esté redactada para despertar la curiosidad de las Universidades del primer mundo por el folklor, la mala sintaxis y la falta de argumentos de esa gente tan especial y primitiva que, al parecer, somos todos nosotros. Quiero decir, la escritura de Villoro se rige por el rigor más estricto y también por el orgullo de un pensador original que se sabe tal y que, por ello, busca el reconocimiento en la insobornable confrontación y la dura crítica, no en los premios patermalistas ni en la compasión.

En segundo lugar, el corpus de la obra de Villoro es también ejemplar en el sentido de que estamos ante una reflexión que ha sido fiel a sí misma sin dejar de haber estado constantemente en conversación con lós mejores pensadores del pasado $y$ del presente. Ese delicado equilibrio entre compromiso con su circunstancia, fidelidad al propio camino y apertura ininterrumpida al otro le ha permitido no sólo evitar el entusiasmo nacionalista, sino también otros vicios opuestos a éste, pero igualmente comunes entre nosotros, como el afán de novedades y el fervor sucursalero.

De ahí que cada nuevo texto de Villoro provoque enseguida la necesidad de lccrlo y también la necesidad de responder críticamente a él. Sus páginas más recientes, El poder y el valor : ilustran lo que estoy diciendo. Son una prolongación de la investigación en teoría del conocimiento comenzada con su admirable libro Creer, saber, conocer, y retoman algunos de los problemas centrales de la moral y la política contemporáneas, en diálogo con la tradición, entre otros, con Maquiavelo, Rousseau y Marx, y a la vez, con el presente, por ejemplo, con John Rawls, Thomas Nagel, Adolfo Sánchez Vázquez y Ernesto Garzón Valdés. Todo ello hace que discutir sus idcas con seriedad nos obligue a elegir algunas de sus líneas temáticas, pues cualquier otra opción equivale a condenarse a vaguedades, indignas de Villoro. 
Después del derrumbe del llamado «socialismo real», la democracia ha regresado a ser el tema central de la política, tanto de la práctica política como de su reflexión, y por supuesto, el tema de la democracia no podía faltar en un libro que se subtitula «Fundamentos de una ética política». En efecto, en la cuarta y última parte de su libro Villoro estudia «la asociación politica» y se pregunta cuál es la asociación política más deseable. Esta pregunta se contesta en relación con el tipo de bien común que persiguen dichas asociaciones. Así, podemos hablar - nos dice Villorode una *asociación para el orden», de una «asociación para la libertad» y de una «asociación para la comunidad».

En relación con la asociación para la libertad, Villoro distingue entre el modelo liberal y el modelo igualitario, agregando que éste no contraviene los fines del primero sino que pretende llevarlos a término (cfr. pp. 314 y ss.):

La realización plena de la asociación para la libertad es, pues, un proyecto por cumplir, una idea regulativa de transformación social (p. 315).

Éste sería el momento en que se podría comenzar una polémica con Villoro y señalar que sólo disponcmos de los dos primeros tipos de asociación y que el tercero sólo se refiere a una desquiciante utopía. Esta objeción se podría respaldar indicando que no puede haber otro bien común en una sociedad plural que la libertad. Quiero decir: el concepto de Aristóteles de «vida buena», de vida buena en común - concepto central para las tradiciones comunitarias que tanto atraen a Villoro-. en los modernos no puede recibir otro contenido concreto que el áspero, a veces angustiante pero inevitable ejercicio de la libertad. Villoro mismo parece aceptar esto cuando defiende:

Todo puede ser objeto de aceptación, limjtación o rechazo excepto los valores que hacen posible esa discusión y fundan los derechos humanos de los interlocutores (p. 321).

Pero dejemos este concepto del bien común, de la vida buena colectiva, para más adelante y vayamos ya al concepto de democracia:

La asociación para el orden podía tomar forma en diferentes sistemas de gobiemo; la asocación para la libertad, en cambio, en cualquiera de sus modelos, ha encontrado su régimen propio en la democracia (p. 333).

Sin embargo, esta unanimidad no esconde que haya por lo menos dos sentidos de democracia: uno "conforme al valor» y otro «conforme al poder». Senala Villoro:

Considerarla como un ideal ético por realizar implica que todos los que intervienen en el acuerdo que da lugar a la asociación convienen en el valor superior de la libertad. Identificarla, en cambio, con las instituciones y prácticas tealmente existentes equivale a verla como el resultado de determinadas relacioncs de poder. Resultaria del acuerdo entre partes en que cada una se rige por su interćs particular. Por el convenio entre ellas se plantearia un bien común a todas: la coexistencia sin violencia excesiva de los intereses opuestos (p. 334).

Concentrándose puramente en el segundo sentido, Schumpeter redujo la democracia a un modus procedendi $\rightarrow$ competencia regulada de individuos y partidos por medio de elecciones-. Pero al perder de vista el primer sentido de democracia, el segundo sentido pierde su justificación, y como indica Villoro, Schumpeter no puede ya decirnos por qué deberíamos preferir ese modus procedendi a otros. En relación con este tipo de conceptos, en otra parte he propuesto hablar de econceptos tensosn: conceptos necesariamente divididos entre un sentido límite y un sentido realizador. El sentido límite nos dice por qué queremos ese concepto y lo justifica; el sentido realizador se refiere a las prácticas 
más adecuadas para alcanzar el sentido límite, aunque reconocicndo que éstas van a ser siempre falibles. Por ejemplo, el concepto de saber es un concepto tenso: su sentido realizador, su modus operandi, es creencia justificada, y su sentido limite cierto modo pertinente de que esa creencia justificada resulte, además, verdadera.

No estoy seguro de si Villoro está de acuerdo conmigo en que el concepto de democracia es un concepto tenso -inevitablemente tenso- $y$ en que tenemos que aprender a vivir críticamente con esta tensión. O si, por el contrario, piensa que su sentido realizador ha sido una horrible traición al sentido límite de democracia: el "poder del pueblo para el pueblo» no ha dejado de transformarse en una forma más de dominio. A veces Villoro parece sugerir esto último cuando enumcra algunos «desvíos del poder del pueblo» (p. 340) como - según él-- la representación, la burocracia y la tecnoctacia y propone una «democracia radical».

Sin embargo, por más «radical» que sea una democracia, ésta nunca dejará, nunca puede dejar de ser un concepto tenso. ¿Cómo es esto? Indica Villoro:

El pueblo real es heterogéneo, está formado por una multiplicidad de comunidades, villas, organizaciones sociales, grupos, etnias y nacionalidades, regiones, estamentos, gremios, confesiones, sectas, federaciones distintas, a veces opuestas, otras entremezcladas. El hombre del pueblo no es un ciudadano abstracto, «alguien» igual a cualquier otro (p. 345).

Pienso que este pasaje está, como diría Vaz Ferreira, «sombreado» por la falsa oposición. Las personas poscemos diversas identidades materiales - diversas pertenencias histórico-sociales-, pero también una identidad formal: somos seres humanos. $Y$ ciertos derechos nos atañen por poseer esas identidades materiales y otros por nuestra identidad formal. Sin el primer reconocimiento no hay posibilidad de una «democracia social». Pero sin el scgundo se desvanece toda idea de «democracia política», de «igualdad política», y así perdemos el concepto mismo de ciudadanía.

Por otra parte, en sus «correcciones a la democracia existente» Villoro propone, entre otras medidas, una «difusión de poderes». Sin embargo, sospecho que es demasiado optimista cuando piensa que pucden coexistir sin constantes y graves conflictos el localismo y el Estado central. Villoro nos dice del poder central:

Tendria la responsabilidad, sobre todo, de la realización de los bienes pủblicos que ningún interés local acertaria a promover, por ejemplo: la investigación científica básica, el uso racional de los recursos naturales del país, la utilización de nuevas fuentes de energía, cl cuidado del medio ambiente y la protección contra el deterioro ecológico. Éstos son valores que responden al interés general de todo hombre (p. 348).

En la última oración, como en muchos otros pasajes de su libro, Villoro reconoce, pues, que además de las identidades materiales hay intereses generales de todo ser humano en tanto ser humano: que hay, pues, una identidad formal. Sin embargo, en relación con este último pasaje me interesaba sobre todo señalar que en cualquiera de los rubros indicados se han producido $y$, previsiblemente, se seguirán produciendo gravísimos conflictos entre los intereses locales y los generales. Villoro comienza su lista con la investigaciôn cientifica. Entre otros, Garzón Valdés ha recordado los inevitables $y$, a veces, trágicos conflictos entre la medicina tradicional y la medicina resultado de la aplicación de la investigación en cicncias naturales. La utilización de nuevas fuentes de energía con frecuencia destruye las formas de vida tradicionales. Y la protección ecológica es sumamente cara y a menudo entra en conflicto con los intereses locales más inmediatos. Sobre todos estos conflictos hay abundante investigación empírica que nos deja concluir que en muchos casos no hay otra solu- 
ción que la subordinación de una de las partes.

También entre sus «correcciones», teniendo en cuenta la corrupción de los partidos políticos y sus no pocas veces fraudulenta representación de los intereses de los votantes, Villoro propone la edemocracia directa" en la forma del referéndum. Pero, se sabe, la historia del referéndum en las socicdades modernas ha sido siempre la historia de un referéndum organizado y dirigido, cuando no manipulado, por los partidos políticos y los medios masivos de comunicación, $y$ estos últimos suelen responder a los intereses generales menos todavía que los primeros. Pero no me imagino de qué otra manera se podría llevar a cabo un referéndum en una socjedad compleja.

Estas observaciones nos regresan a la comprobación de que la democracia es de manera inevitable un concepto tenso: que constantemente el sentido realizador, el modus operandi, se «desviarà y hasta se pervertirá y que no puede haber ni correcciones estables ni arreglos institucionales infalibles: sólo el cjercicio de caso en caso de la libertad y sus productos, la indignación moral y el pensamiento crítico podrá cvitar que la inevitable tensión se convierta en traición.

Quiero todavía regresar un poco a la polémica ya anunciada respecto del contenido de «asociación para la comunidad». Dice Villoro:

Una asociación es conforme al valor cuando hace coincidir los intereses particulares de sus miembros con el interés general. Cuando esa coincidencia es cabal y cada quién vela por el bien del todo de la misma manera que por su bien personal, cuando todos los sujetos de una colectividad incluyen en su deseo to deseable para el todo, entonces no hay distinción entre el bien común y el bien individual; la asociación se ha convertido en una comunidad (p. 359).

Descreo de todo ello. Sospecho que es una peligrosa ilusión pensar que es posible que, de manera sistemática y estable, desaparezca la distinción entre el bien común y el bien individual, y si ésa es la condición para que haya comunidad, entonces nunca genuinamente la hay. En una sociedad plural -y por lo menos todas las sociedades no tradicionales lo son- no es eliminable el conflicto constante entre deseos y creencias de sus micmbros más que apelando al terror. La educación para la disidencia, como nos he enseñado Javier Muguerza, es parte de cualquier educación democrática. De ahí que insista: en los modernos el único contenido del bien común de la vida buena es la libertad.

Por eso, también discrepo con Villora cuando señala:

La comunidad está presente como limite posible en toda asociación conforme al valor (p. 360).

De nuevo, no lo creo. Por el contrario, pienso que este concepto de sasociación para la comunidad", como ya adelanté, no designa ideales valiosos -el sentido límite que debe realizar toda asociación conforIne al valor -, sino -otra vez- peligrosas ilusiones. ¿Por qué afirmo tal cosa? Pues porque quien en política - como en general en la vida - se aferra a deseos imposibles se condena a desechar los muchos deseos posibles cuya realización enriquecen la vida tanto privada como pública. Por ejemplo, en la historia reciente a veces se le ha pedido tanto a las «asociaciones conforme al valor» que no se ha sabido apreciar lo mucho que ellas realmente pueden darnos. Recuerdo las críticas de algunos marxistas a la «imperfecta» democracia «formal», críticas que en los setenta colaboraron a sumir a Amériça Latina en los más atroces regímenes autoritarios, muchos de ellos incluso saludados - al comienzo al menos- «como csperanzas de algo propio, de algo nuevo\%.

Villoro no ignora estos contra-argumentos y por eso indica que la comunidad puede justificarse: 
en la repetición de una costumbre o en la libertad. Un servicio puede ser impuesto, pero también elegido libremente; puede consistir en una afirmación de una voluntad ajena o en una realización de sí mismo (p. 360).

Pero se le podría replicar a Villoro: la libertad nunca se puede donar más que en grados, so pena de perderse. Una libertad que se enajena totalmente y para siempre - en la política, en el amor...- se pervierte, deja de ser tal. Algo de esto reconoce el mismo Villoro cuando afirma:

El servicio no implica necesariamente pérdida de autonomia (p. 361),

Mấs todavía, yo agregaría que cuando la implica se transforma en ciega servidumbre o en adicción enloquecida: y ambas son, se sabe, máscaras del miedo.

La fratemidad, el sentido de la cormunidad, las fuerzas del amor configuran potencialidades valiosísimas que poseen las personas y que a menudo encuentran realización en muchas interacciones individuales y sociales. Pero no son el tipo de valores que puede regir la vida en común de una sociedad compleja y, por tanto, llena de conflictos entre los deseos y creencias de sus miembros. Al respecto, la historia de este siglo atroz nos ha cnseñado que con frecuencia menos es más.

Confieso que al releer el libro de Villoro y estas mis apresuradas notas no estoy seguro de si con ellas he elaborado fuertes ataques a algunos de los importantes argumentos de Villoro o sólo comentarios, con algún que otro matiz de tono, que por qujzá turbias razones polémicas he sobreacentuado. En cualquier caso, quien repiense estos problemas, o cualquiera de los otros de este libro excepcional, no hara más que confirmar que sin duda nos encontramos ante uno de nuestros grandes maestros, e incluso algo más: ante uno de nuestros maestros definitivamente imprescindibles. 


\title{
T H E O R I A \\ REVISTA DE TEORIA, HISTORIA Y FUNDAMENTOS DE LA CIENCIA \\ FUNDADA EN 1952 - SEGUNDA EPOCA \\ FUNDADOR: Miguel SANCHEZ-MAZAS ( $\dagger$ ) \\ Revista asociada a la Sociedad de Lógica, Metodologia y Filosoffa de la Ciencia en España y a la Sociedad Espaniola de Filosofia Analitica
}

Vol. 14

Enero / January

$1999 / 1$

\section{SUMARIO/ CONTENTS}

\author{
SECCION MONOGRAFICA: \\ Analytical Pbilosophy and Epistemology in Ukraine \\ Guest Editors: Yaroslav SHRAMKO, Olga KORPALO \\ Olga KORPALO (Kiev), \\ Valentin OMELYANTCHIK (Kiev), \\ Yaroslay SHRAMKO (Krivoi Rog) \\ Andriy VASYLCHENKO (Kiev) \\ Valentin OMELYANTCHIK (Kiev) \\ Vladimir KUZNETSOV (Kiev) \\ Yaroslav SHRAMKO (Krivoi Rog) \\ Volodymyr NAVROTSKYY (Kharkov) \\ Anatoliy ISHMURATOV (Kiev) \\ Olga KORPALO (Kiev)

\section{5-9 Presentation} \\ 11-24 Apprehensio Simplex in the Kiew-Molyla Arademy \\ 25-38 Aristotle's ExtensionalModality: Hintikka's Intuitions, \\ Lukasiewicz's Logic and Mignucci's Verdict \\ 39-62 On the Triplet Frame for Concept Analysis \\ 63-81 A Theory of Relevant Properties 1: Reflections and \\ Definitions \\ 83-94 Paraconsistent Description of Change \\ 95-107 Logical Modelling of Conflict Phenomenon \\ 109-127 Rationality and Emotions (The Perspectives of Logical- \\ Cognitive Analysis)
}

\section{ARTICULOS / ARTICLES}

Matthias SCHIRN (Munich) 131-156 Sobre la teoria fregeona de las oraciones no extensionales (On Frege's Theory of Non-Extensional Sentences)

José A. DIEZ (Tarragona) 157-178 A General Representation for Internal Proportional Combinatorial Medsurement Systems when the Operation Is Not Necessarily Closed

\begin{abstract}
RECENSIONES Y LIBROS RECIBIDOS / BOOK REVEWS AND BOOKS RECEIVED CRONICAS Y PROXIMAS REUNIONES / NOTICES AND ANNOUNCEMENTS SUMARIO ANALITICO / SUMMARY
\end{abstract}

\section{REDACCION / EDITORLAL OFFICE}

ESPANAASPAIN: CALIJ-THEORIA, Alcalde José Eloscgui, 275, E 20015, Apartado 1.594, 20080 San Sebastiăn, España.

Tel.: (34 43) 29.17.25. Fax (A. Ibarra): (34 43) 31.10.56. E-mail: theoria@sf.ehu.es

FXTRANIERO FOREIGN COUNTRIES: Asociación Cultural España-THEORIA, Case 2.730, 1211 Genève-2, Suisse.

\section{DISTRIBUCION / DISTRIBUTION}

Para suscripciones, numeros atrasados y cambios de dirección: $/$ For subscriptions, back volumes and changes of addrcy: Servicio Editorial, Universidad del Pás Vasco, Apartado 1.397, E 48080 Leioa, Espania Tel. (34 4) 464.77.00 (ext. 2153). Fax: (34 4) 480.13.14. E-mail: luxediro@lg, ehu.es THEORLA es una revista cuatrimestral (sale en Enero. Mayo y Septiembre) / THEORLA is a four-menthy joutnal (istwer in january, May and September) 